\title{
GENERALIZED ORDER $(\alpha, \beta)$ ORIENTED SOME GROWTH PROPERTIES OF COMPOSITE ENTIRE FUNCTIONS
}

\author{
Tanmay Biswas \\ Rajbari, Rabindrapally, \\ R.N. Tagore Road, P.O.-Krishnagar, Dist-Nadia, PIN-741101, West Bengal, India \\ tanmaybiswas_math@rediffmail.com

\section{Chinmay Biswas} \\ Department of Mathematics, Nabadwip Vidyasagar College, \\ Nabadwip, Dist.-Nadia, PIN-741302, West Bengal, India \\ chinmay.shib@gmail.com

\begin{abstract}
In this paper we establish some results relating to the growths of composition of two entire functions with their corresponding left and right factors on the basis of their generalized order $(\alpha, \beta)$ and generalized lower order $(\alpha, \beta)$ where $\alpha$ and $\beta$ are continuous non-negative functions on $(-\infty,+\infty)$.
\end{abstract}

Keywords: Entire function, Growth, Composition, Generalized order $(\alpha, \beta)$, Generalized lower order $(\alpha, \beta)$.

\section{Introduction, definitions and notations}

We denote by $\mathbb{C}$ the set of all finite complex numbers. Let $f$ be an entire function defined on $\mathbb{C}$. The maximum modulus function $M_{f}(r)$ and the maximum term $\mu_{f}(r)$ of

$$
f=\sum_{n=0}^{\infty} a_{n} z^{n}
$$

on $|z|=r$ are defined as

$$
M_{f}=\max _{|z|=r}|f(z)|, \quad \mu_{f}(r)=\max _{n \geq 0}\left(\left|a_{n}\right| r^{n}\right)
$$

respectively. We use the standard notations and definitions of the theory of entire functions which are available in [11] and [12], and therefore we do not explain those in details. For $x \in[0, \infty)$ and $k \in \mathbb{N}$ where $\mathbb{N}$ be the set of all positive integers, define iterations of the exponential and logarithmic functions as

$$
\exp ^{[k]} x=\exp \left(\exp ^{[k-1]} x\right), \quad \log ^{[k]} x=\log \left(\log ^{[k-1]} x\right),
$$

with convention that

$$
\log ^{[0]} x=x, \quad \log ^{[-1]} x=\exp x, \quad \exp ^{[0]} x=x, \quad \exp ^{[-1]} x=\log x .
$$

Now considering this, let us recall that Juneja et al. [5] defined the $(p, q)$-th order and $(p, q)$-th lower order of an entire function, respectively, as follows: 
Definition 1 [5]. The $(p, q)$-th order and $(p, q)$-th lower order of an entire function $f$ are defined as:

$$
\begin{aligned}
& \rho^{(p, q)}(f) \\
& \lambda^{(p, q)}(f)
\end{aligned}=\lim _{r \rightarrow+\infty} \sup \frac{\log ^{[p]} M_{f}(r)}{\log ^{[q]} r},
$$

where $p$ and $q$ always denote positive integers with $p \geq q$.

Extending the notion $(p, q)$-th order, recently Shen et al. [6] introduced the new concept of $[p, q]-\varphi$ order of an entire function where $p \geq q$. Later on, combining the definition of $(p, q)$ order and $[p, q]-\varphi$ order, Biswas (see, e.g., [1]) redefined the $(p, q)$-order of an entire function without restriction $p \geq q$.

However the above definition is very useful for measuring the growth of entire functions. If $p=l$ and $q=1$ then we write $\rho^{(l, 1)}(f)=\rho^{(l)}(f)$ and $\lambda^{(l, 1)}(f)=\lambda^{(l)}(f)$ where $\rho^{(l)}(f)$ and $\lambda^{(l)}(f)$ are respectively known as generalized order and generalized lower order of entire function $f$. For details about generalized order one may see [8]. Also for $p=2$ and $q=1$, we respectively denote $\rho^{(2,1)}(f)$ and $\lambda^{(2,1)}(f)$ by $\rho(f)$ and $\lambda(f)$ which are classical growth indicators such as order and lower order of entire function $f$. Recently, Chyzhykov et al. [3] showed that both the definitions of generalized order and $(p, q)$-order have the disadvantage that they do not cover arbitrary growth (see [3, Example 1.4]).

Taking this into account, let $L$ be a class of continuous non-negative on $(-\infty,+\infty)$ function $\alpha$ such that

$$
\alpha(x)=\alpha\left(x_{0}\right) \geq 0, \quad \text { for } \quad x \leq x_{0} \quad \text { with } \quad \alpha(x) \uparrow+\infty \quad \text { as } \quad x \rightarrow+\infty
$$

and

$$
\alpha((1+o(1)) x)=(1+o(1)) \alpha(x) \quad \text { as } \quad x \rightarrow+\infty .
$$

We say that $\alpha \in L^{0}$, if $\alpha \in L$ and

$$
\alpha(c x)=(1+o(1)) \alpha(x) \quad \text { as } \quad x_{0} \leq x \rightarrow+\infty
$$

for each $c \in(0,+\infty)$, i.e., $\alpha$ is slowly increasing function. Clearly $L^{0} \subset L$.

Further we assume that throughout the present paper $\alpha, \alpha_{1}, \alpha_{2}, \beta, \beta_{1}$ and $\beta_{2}$ always denote the functions belonging to $L^{0}$.

Considering this, the value

$$
\rho_{(\alpha, \beta)}[f]=\limsup _{r \rightarrow+\infty} \frac{\alpha\left(\log M_{f}(r)\right)}{\beta(\log r)} \quad(\alpha \in L, \quad \beta \in L)
$$

is called [7] the generalized order $(\alpha, \beta)$ of an entire function $f$. For details about the generalized order $(\alpha, \beta)$ one may see [7]. During the past decades, several authors made close investigations on the properties of entire functions related to the generalized order $(\alpha, \beta)$ in some different direction. For the purpose of further applications, Biswas et al. [2] rewrite the definition of the generalized order $(\alpha, \beta)$ of entire function in the following way after giving a minor modification to the original definition (e.g. see, [7]) which considerably extend the definition of $\varphi$-order of entire function introduced by Chyzhykov et al. [3]:

Definition 2 [2]. The generalized order $(\alpha, \beta)$ and the generalized lower order $(\alpha, \beta)$ of an entire function $f$ are defined as:

$$
\begin{aligned}
& \rho_{(\alpha, \beta)}[f] \\
& \lambda_{(\alpha, \beta)}[f]
\end{aligned}=\lim _{r \rightarrow+\infty} \sup \frac{\alpha\left(M_{f}(r)\right)}{\beta(r)} .
$$


Definition 1 is a special case of Definition 2 for $\alpha(r)=\log ^{[p]} r$ and $\beta(r)=\log ^{[q]} r$.

Since for $0 \leq r<R$,

$$
\mu_{f}(r) \leq M_{f}(r) \leq \frac{R}{R-r} \mu_{f}(R) \quad(\text { cf. [10]) }
$$

it is easy to see that

$$
\left.\begin{array}{l}
\rho_{(\alpha, \beta)}[f] \\
\lambda_{(\alpha, \beta)}[f]
\end{array}=\lim _{r \rightarrow+\infty} \sup _{\inf } \frac{\alpha\left(\mu_{f}(r)\right)}{\beta(r)} \quad \text { (also see }[2]\right) .
$$

In the paper we would like to establish some newly developed results based on the comparative growth of composite entire functions on the basis of their generalized order $(\alpha, \beta)$ and generalized lower order $(\alpha, \beta)$.

\section{Known results}

In this section we present some lemmas which will be needed in the sequel.

Lemma 1 [4]. Let $f$ and $g$ are any two entire functions with $g(0)=0$. Also let $b$ satisfy $0<b<1$ and $c(b)=(1-b)^{2} /(4 b)$. Then for all sufficiently large values of $r$, we have

$$
M_{f}\left(c(b) M_{g}(b r)\right) \leq M_{f \circ g}(r) \leq M_{f}\left(M_{g}(r)\right) .
$$

In addition if $b=1 / 2$, then for all sufficiently large values of $r$, the inequality is true

$$
M_{f \circ g}(r) \geq M_{f}\left(\frac{1}{8} M_{g}\left(\frac{r}{2}\right)\right) .
$$

Lemma 2 [9]. Let $f$ and $g$ be entire functions. Then for every $\delta>1$ and $0<r<R$, we have

$$
\mu_{f \circ g}(r) \leq \frac{\delta}{\delta-1} \mu_{f}\left(\frac{\delta R}{R-r} \mu_{g}(R)\right) .
$$

Lemma 3 [9]. If $f$ and $g$ are any two entire functions. Then for all sufficiently large values of $r$, the estimate is true

$$
\mu_{f \circ g}(r) \geq \frac{1}{2} \mu_{f}\left(\frac{1}{16} \mu_{g}\left(\frac{r}{4}\right)\right)
$$

\section{Main results}

In this section we present the main results of the paper.

Theorem 1. Let $f$ and $g$ be any two entire functions such that

$$
0<\lambda_{\left(\alpha_{1}, \beta_{1}\right)}[f] \leq \rho_{\left(\alpha_{1}, \beta_{1}\right)}[f]<+\infty, \quad \text { and } \quad \rho_{\left(\alpha_{2}, \beta_{2}\right)}[g]>0 .
$$

If $\alpha_{2}\left(\beta_{1}^{-1}(r)\right) \in L_{1}$, then we have the estimate

$$
\limsup _{r \rightarrow+\infty} \frac{\alpha_{2}\left(\beta_{1}^{-1}\left(\alpha_{1}\left(\mu_{f \circ g}\left(\beta_{2}^{-1}(r)\right)\right)\right)\right)}{\alpha_{1}\left(\mu_{f}\left(\beta_{1}^{-1}(r)\right)\right)} \geq \frac{\rho_{\left(\alpha_{2}, \beta_{2}\right)}[g]}{\rho_{\left(\alpha_{1}, \beta_{1}\right)}[f]} .
$$


P r o o f. From the definition of $\rho_{\left(\alpha_{1}, \beta_{1}\right)}[f]$, we get for all sufficiently large values of $r$ that

$$
\alpha_{1}\left(\mu_{f}\left(\beta_{1}^{-1}(r)\right)\right) \leq\left(\rho_{\left(\alpha_{1}, \beta_{1}\right)}[f]+\varepsilon\right) r .
$$

Further in view of the first part of Lemma 3, it follows for all sufficiently large values of $r$ that

$$
\alpha_{1}\left(\mu_{f \circ g}\left(\beta_{2}^{-1}(r)\right)\right) \geq(1+o(1))\left(\lambda_{\left(\alpha_{1}, \beta_{1}\right)}[f]-\varepsilon\right) \beta_{1}\left(\mu_{g}\left(\frac{\beta_{2}^{-1}(r)}{4}\right)\right) .
$$

Since $\alpha_{2}\left(\beta_{1}^{-1}(r)\right) \in L_{1}$, we obtain from above for a sequence of values of $r$ tending to infinity that

$$
\begin{aligned}
\alpha_{2}\left(\beta_{1}^{-1}\left(\alpha_{1}\left(\mu_{f \circ g}\left(\beta_{2}^{-1}(r)\right)\right)\right)\right) & \geq(1+o(1)) \alpha_{2}\left(\mu_{g}\left(\frac{\beta_{2}^{-1}(r)}{4}\right)\right) \\
i . e ., \alpha_{2}\left(\beta_{1}^{-1}\left(\alpha_{1}\left(\mu_{f \circ g}\left(\beta_{2}^{-1}(r)\right)\right)\right)\right) & \geq(1+o(1))\left(\rho_{\left(\alpha_{2}, \beta_{2}\right)}[g]-\varepsilon\right) r .
\end{aligned}
$$

Now combining (3.1) and above inequalities we get that

$$
\limsup _{r \rightarrow+\infty} \frac{\alpha_{2}\left(\beta_{1}^{-1}\left(\alpha_{1}\left(\mu_{f \circ g}\left(\beta_{2}^{-1}(r)\right)\right)\right)\right)}{\alpha_{1}\left(\mu_{f}\left(\beta_{1}^{-1}(r)\right)\right)} \geq \frac{\rho_{\left(\alpha_{2}, \beta_{2}\right)}[g]}{\rho_{\left(\alpha_{1}, \beta_{1}\right)}[f]} .
$$

Hence the theorem follows.

Theorem 2. Let $f$ and $g$ be any two entire functions such that

$$
0<\lambda_{\left(\alpha_{1}, \beta_{1}\right)}[f] \leq \rho_{\left(\alpha_{1}, \beta_{1}\right)}[f]<+\infty \quad \text { and } \quad \lambda_{\left(\alpha_{2}, \beta_{2}\right)}[g]>0 .
$$

If $\alpha_{2}\left(\beta_{1}^{-1}(r)\right) \in L_{1}$, then

$$
\liminf _{r \rightarrow+\infty} \frac{\alpha_{2}\left(\beta_{1}^{-1}\left(\alpha_{1}\left(\mu_{f \circ g}\left(\beta_{2}^{-1}(r)\right)\right)\right)\right)}{\alpha_{1}\left(\mu_{f}\left(\beta_{1}^{-1}(r)\right)\right)} \geq \frac{\lambda_{\left(\alpha_{2}, \beta_{2}\right)}[g]}{\rho_{\left(\alpha_{1}, \beta_{1}\right)}[f]} .
$$

Theorem 3. Let $f$ and $g$ be any two entire functions such that

$$
0<\lambda_{\left(\alpha_{1}, \beta_{1}\right)}[f]<+\infty \quad \text { and } \quad \lambda_{\left(\alpha_{2}, \beta_{2}\right)}[g]>0 .
$$

If $\alpha_{2}\left(\beta_{1}^{-1}(r)\right) \in L_{1}$, then

$$
\limsup _{r \rightarrow+\infty} \frac{\alpha_{2}\left(\beta_{1}^{-1}\left(\alpha_{1}\left(\mu_{f \circ g}\left(\beta_{2}^{-1}(r)\right)\right)\right)\right)}{\alpha_{1}\left(\mu_{f}\left(\beta_{1}^{-1}(r)\right)\right)} \geq \frac{\lambda_{\left(\alpha_{2}, \beta_{2}\right)}[g]}{\lambda_{\left(\alpha_{1}, \beta_{1}\right)}[f]} .
$$

The proofs of Theorem 2 and Theorem 3 would run parallel to that of Theorem 1 . We omit the details.

Theorem 4. Let $f$ and $g$ be any two entire functions such that

$$
0<\lambda_{\left(\alpha_{1}, \beta_{1}\right)}[f] \leq \rho_{\left(\alpha_{1}, \beta_{1}\right)}[f]<+\infty \quad \text { and } \quad \rho_{\left(\alpha_{2}, \beta_{2}\right)}[g]<+\infty .
$$

If $\alpha_{2}\left(\beta_{1}^{-1}(r)\right) \in L_{1}$, then

$$
\limsup _{r \rightarrow+\infty} \frac{\alpha_{2}\left(\beta_{1}^{-1}\left(\alpha_{1}\left(\mu_{f \circ g}\left(\beta_{2}^{-1}(r)\right)\right)\right)\right)}{\alpha_{1}\left(\mu_{f}\left(\beta_{1}^{-1}(r)\right)\right)} \leq \frac{\rho_{\left(\alpha_{2}, \beta_{2}\right)}[g]}{\lambda_{\left(\alpha_{1}, \beta_{1}\right)}[f]} .
$$


P r o o f. From the definition of $\lambda_{\left(\alpha_{1}, \beta_{1}\right)}[f]$, we get for all sufficiently large values of $r$ that

$$
\alpha_{1}\left(\mu_{f}\left(\beta_{1}^{-1}(r)\right)\right) \geq\left(\lambda_{\left(\alpha_{1}, \beta_{1}\right)}[f]-\varepsilon\right) r .
$$

Further taking $R=2 r$ in Lemma 2 we obtain for all sufficiently large values of $r$ that

$$
\alpha_{1}\left(\mu_{f \circ g}(r)\right) \leq(1+o(1))\left(\rho_{\left(\alpha_{1}, \beta_{1}\right)}[f]+\varepsilon\right) \beta_{1}\left(\mu_{g}(2 r)\right) .
$$

Since $\alpha_{2}\left(\beta_{1}^{-1}(r)\right) \in L_{1}$, we obtain from above for all sufficiently large values of $r$ that

$$
\begin{aligned}
\alpha_{2}\left(\beta_{1}^{-1}\left(\alpha_{1}\left(\mu_{f \circ g}\left(\beta_{2}^{-1}(r)\right)\right)\right) g\right) & \leq(1+o(1)) \alpha_{2}\left(\mu_{g}\left(2 \beta_{2}^{-1}(r)\right)\right) \\
i . e ., \alpha_{2}\left(\beta_{1}^{-1}\left(\alpha_{1}\left(\mu_{f \circ g}\left(\beta_{2}^{-1}(r)\right)\right)\right)\right) & \leq(1+o(1))\left(\rho_{\left(\alpha_{2}, \beta_{2}\right)}[g]+\varepsilon\right) r .
\end{aligned}
$$

Now combining (3.2) and above inequalities we get that

$$
\limsup _{r \rightarrow+\infty} \frac{\alpha_{2}\left(\beta_{1}^{-1}\left(\alpha_{1}\left(\mu_{f \circ g}\left(\beta_{2}^{-1}(r)\right)\right)\right)\right)}{\alpha_{1}\left(\mu_{f}\left(\beta_{1}^{-1}(r)\right)\right)} \leq \frac{\rho_{\left(\alpha_{2}, \beta_{2}\right)}[g]}{\lambda_{\left(\alpha_{1}, \beta_{1}\right)}[f]} .
$$

Hence the theorem follows.

Theorem 5. Let $f$ and $g$ be any two entire functions such that

$$
0<\lambda_{\left(\alpha_{1}, \beta_{1}\right)}[f] \leq \rho_{\left(\alpha_{1}, \beta_{1}\right)}[f]<+\infty \quad \text { and } \quad \lambda_{\left(\alpha_{2}, \beta_{2}\right)}[g]<+\infty .
$$

If $\alpha_{2}\left(\beta_{1}^{-1}(r)\right) \in L_{1}$, then we have

$$
\liminf _{r \rightarrow+\infty} \frac{\alpha_{2}\left(\beta_{1}^{-1}\left(\alpha_{1}\left(\mu_{f \circ g}\left(\beta_{2}^{-1}(r)\right)\right)\right)\right)}{\alpha_{1}\left(\mu_{f}\left(\beta_{1}^{-1}(r)\right)\right)} \leq \frac{\lambda_{\left(\alpha_{2}, \beta_{2}\right)}[g]}{\lambda_{\left(\alpha_{1}, \beta_{1}\right)}[f]}
$$

Theorem 6. Let $f$ and $g$ be any two entire functions such that

$$
0<\lambda_{\left(\alpha_{1}, \beta_{1}\right)}[f] \leq \rho_{\left(\alpha_{1}, \beta_{1}\right)}[f]<+\infty \quad \text { and } \quad \rho_{\left(\alpha_{2}, \beta_{2}\right)}[g]<+\infty .
$$

If $\alpha_{2}\left(\beta_{1}^{-1}(r)\right) \in L_{1}$, then we have

$$
\liminf _{r \rightarrow+\infty} \frac{\alpha_{2}\left(\beta_{1}^{-1}\left(\alpha_{1}\left(\mu_{f \circ g}\left(\beta_{2}^{-1}(r)\right)\right)\right)\right)}{\alpha_{1}\left(\mu_{f}\left(\beta_{1}^{-1}(r)\right)\right)} \leq \frac{\rho_{\left(\alpha_{2}, \beta_{2}\right)}[g]}{\rho_{\left(\alpha_{1}, \beta_{1}\right)}[f]} .
$$

The proofs of Theorem 5 and Theorem 6 would run parallel to that of Theorem 4 . We omit the details.

Theorem 7. Let $f, g, h$ and $k$ be four entire functions such that

$$
\lambda_{\left(\alpha_{3}, \beta_{3}\right)}[h]>0, \quad \lambda_{\left(\alpha_{4}, \beta_{4}\right)}[k]>0 \quad \text { and } \quad \rho_{\left(\alpha_{2}, \beta_{2}\right)}[g]<\lambda_{\left(\alpha_{4}, \beta_{4}\right)}[k] .
$$

Also let $C$ and $D$ be any two positive constants.

(i) Any one of the following four conditions are assumed to be satisfied:

(a) $\beta_{1}(r)=C\left(\exp \left(\alpha_{2}(r)\right)\right)$ and $\beta_{3}(r)=D \exp \left(\alpha_{4}(r)\right)$;

(b) $\beta_{1}(r)=C\left(\exp \left(\alpha_{2}(r)\right)\right)$ and $\beta_{3}(r)>\exp \left(\alpha_{4}(r)\right)$;

(c) $\exp \left(\alpha_{2}(r)\right)>\beta_{1}(r)$ and $\beta_{3}(r)=D \exp \left(\alpha_{4}(r)\right)$;

(d) $\exp \left(\alpha_{2}(r)\right)>\beta_{1}(r)$ and $\beta_{3}(r)>\exp \left(\alpha_{4}(r)\right)$; 
then

$$
\lim _{r \rightarrow+\infty} \frac{\alpha_{3}\left(\mu_{h \circ k}\left(\beta_{4}^{-1}(\log r)\right)\right)}{\alpha_{1}\left(\mu_{f \circ g}\left(\beta_{2}^{-1}(\log r)\right)\right)}=\infty
$$

(ii) Any one of the following two conditions are assumed to be satisfied:

(a) $\beta_{1}(r)=C\left(\exp \left(\alpha_{2}(r)\right)\right)$ and $\alpha_{4}\left(\beta_{3}^{-1}(r)\right) \in L^{0}$;

(b) $\beta_{3}(r)>\exp \left(\alpha_{4}(r)\right)$ and $\alpha_{4}\left(\beta_{3}^{-1}(r)\right) \in L^{0}$;

then

$$
\lim _{r \rightarrow+\infty} \frac{\exp \left(\alpha_{4}\left(\beta_{3}^{-1}\left(\alpha_{3}\left(\mu_{h \circ k}\left(\beta_{4}^{-1}(\log r)\right)\right)\right)\right)\right.}{\alpha_{1}\left(\mu_{f \circ g}\left(\beta_{2}^{-1}(\log r)\right)\right)}=\infty .
$$

(iii) Any one of the following two conditions are assumed to be satisfied:

(a) $\beta_{3}(r)=D \exp \left(\alpha_{4}(r)\right)$ and $\alpha_{2}\left(\beta_{1}^{-1}(r)\right) \in L^{0}$;

(b) $\beta_{3}(r)>\exp \left(\alpha_{4}(r)\right)$ and $\alpha_{2}\left(\beta_{1}^{-1}(r)\right) \in L^{0}$;

then

$$
\lim _{r \rightarrow+\infty} \frac{\alpha_{3}\left(\mu_{h \circ k}\left(\beta_{4}^{-1}(\log r)\right)\right)}{\exp \left(\alpha_{2}\left(\beta_{1}^{-1}\left(\alpha_{1}\left(\mu_{f \circ g}\left(\beta_{2}^{-1}(\log r)\right)\right)\right)\right)\right.}=\infty .
$$

(iv) If $\alpha_{2}\left(\beta_{1}^{-1}(r)\right) \in L_{1}$ and $\alpha_{4}\left(\beta_{3}^{-1}(r)\right) \in L^{0}$, then

$$
\lim _{r \rightarrow+\infty} \frac{\exp \left(\alpha_{4}\left(\beta_{3}^{-1}\left(\alpha_{3}\left(\mu_{h \circ k}\left(\beta_{4}^{-1}(\log r)\right)\right)\right)\right)\right.}{\exp \left(\alpha_{2}\left(\beta_{1}^{-1}\left(\alpha_{1}\left(\mu_{f \circ g}\left(\beta_{2}^{-1}(\log r)\right)\right)\right)\right)\right.}=\infty .
$$

P r o o f. In view of (3.3) we get for all sufficiently large values of $r$ that

$$
\alpha_{1}\left(\mu_{f \circ g}\left(\beta_{2}^{-1}(\log r)\right)\right) \leq(1+o(1))\left(\rho_{\left(\alpha_{1}, \beta_{1}\right)}[f]+\varepsilon\right) \beta_{1}\left(\mu_{g}\left(2 \beta_{2}^{-1}(\log r)\right)\right) .
$$

Case I. Let $\beta_{1}(r)=C\left(\exp \left(\alpha_{2}(r)\right)\right)$. Then we have from (3.4) for all sufficiently large values of $r$ that

$$
\alpha_{1}\left(\mu_{f \circ g}\left(\beta_{2}^{-1}(\log r)\right)\right) \leq C(1+o(1))\left(\rho_{\left(\alpha_{1}, \beta_{1}\right)}[f]+\varepsilon\right) r^{(1+o(1))\left(\rho_{\left(\alpha_{2}, \beta_{2}\right)}[g]+\varepsilon\right)} .
$$

Case II. Let $\exp \left(\alpha_{2}(r)\right)>\beta_{1}(r)$. Then we have from (3.4) for all sufficiently large values of $r$ that

$$
\alpha_{1}\left(\mu_{f \circ g}\left(\beta_{2}^{-1}(\log r)\right)\right) \leq(1+o(1))\left(\rho_{\left(\alpha_{1}, \beta_{1}\right)}[f]+\varepsilon\right) r^{(1+o(1))\left(\rho_{\left(\alpha_{2}, \beta_{2}\right)}[g]+\varepsilon\right)} .
$$

Case III. Let $\alpha_{2}\left(\beta_{1}^{-1}(r)\right) \in L^{0}$. Then we get from(3.4) for all sufficiently large values of $r$ that

$$
\exp \left(\alpha_{2}\left(\beta_{1}^{-1}\left(\alpha_{1}\left(\mu_{f \circ g}\left(\beta_{2}^{-1}(\log r)\right)\right)\right)\right)\right) \leq r^{(1+o(1))\left(\rho_{\left(\alpha_{2}, \beta_{2}\right)}[g]+\varepsilon\right)} .
$$

Further it follows from Lemma 3 for all sufficiently large values $r$ that

$$
\begin{aligned}
\alpha_{3}\left(\mu_{h \circ k}\left(\beta_{4}^{-1}(\log r)\right)\right) & \geq(1+o(1)) \alpha_{3}\left(\mu_{h}\left(\frac{1}{16} \mu_{k}\left(\frac{\beta_{4}^{-1}(\log r)}{4}\right)\right)\right) \\
\text { i.e., } \alpha_{3}\left(\mu_{h \circ k}\left(\beta_{4}^{-1}(\log r)\right)\right) & \geq(1+o(1))\left(\lambda_{\left(\alpha_{3}, \beta_{3}\right)}[h]-\varepsilon\right) \beta_{3}\left(\mu_{k}\left(\frac{\beta_{4}^{-1}(\log r)}{4}\right)\right) .
\end{aligned}
$$

Case IV. Let $\beta_{3}(r)=D \exp \left(\alpha_{4}(r)\right)$. Then from (3.8) it follows for all sufficiently large values of $r$ that

$$
\alpha_{3}\left(\mu_{h \circ k}\left(\beta_{4}^{-1}(\log r)\right)\right) \geq D(1+o(1))\left(\lambda_{\left(\alpha_{3}, \beta_{3}\right)}[h]-\varepsilon\right) r^{(1+o(1))\left(\lambda_{\left(\alpha_{4}, \beta_{4}\right)}[k]-\varepsilon\right)} .
$$


Case V. Let $\beta_{3}(r)>\exp \left(\alpha_{4}(r)\right)$. Now from (3.8) it follows for all sufficiently large values of $r$ that

$$
\alpha_{3}\left(\mu_{h \circ k}\left(\beta_{4}^{-1}(\log r)\right)\right)>(1+o(1))\left(\lambda_{\left(\alpha_{3}, \beta_{3}\right)}[h]-\varepsilon\right) r^{(1+o(1))\left(\lambda_{\left(\alpha_{4}, \beta_{4}\right)}[k]-\varepsilon\right)} .
$$

Case VI. Let $\alpha_{4}\left(\beta_{3}^{-1}(r)\right) \in L^{0}$. Then from (3.8) we obtain for all sufficiently large values of $r$ that

$$
\exp \left(\alpha_{4}\left(\beta_{3}^{-1}\left(\alpha_{3}\left(\mu_{h \circ k}\left(\beta_{4}^{-1}(\log r)\right)\right)\right)\right)\right) \geq r^{(1+o(1))\left(\lambda_{\left(\alpha_{4}, \beta_{4}\right)}[k]-\varepsilon\right)} .
$$

Since $\rho_{\left(\alpha_{2}, \beta_{2}\right)}[g]<\lambda_{\left(\alpha_{4}, \beta_{4}\right)}[k]$ we can choose $\varepsilon(>0)$ in such a way that

$$
\rho_{\left(\alpha_{2}, \beta_{2}\right)}[g]+\varepsilon<\lambda_{\left(\alpha_{4}, \beta_{4}\right)}[k]-\varepsilon .
$$

Now combining (3.5) of Case I and (3.9) of Case IV it follows for all sufficiently large values of $r$ that

$$
\frac{\alpha_{3}\left(\mu_{h \circ k}\left(\beta_{4}^{-1}(\log r)\right)\right)}{\alpha_{1}\left(\mu_{f \circ g}\left(\beta_{2}^{-1}(\log r)\right)\right)} \geq \frac{D(1+o(1))\left(\lambda_{\left(\alpha_{3}, \beta_{3}\right)}[h]-\varepsilon\right) r^{(1+o(1))\left(\lambda_{\left(\alpha_{4}, \beta_{4}\right)}[k]-\varepsilon\right)}}{C(1+o(1))\left(\rho_{\left(\alpha_{1}, \beta_{1}\right)}[f]+\varepsilon\right) r^{(1+o(1))\left(\rho_{\left(\alpha_{2}, \beta_{2}\right)}[g]+\varepsilon\right)}} .
$$

So from (3.12) and above we obtain that

$$
\liminf _{r \rightarrow+\infty} \frac{\alpha_{3}\left(\mu_{h \circ k}\left(\beta_{4}^{-1}(\log r)\right)\right)}{\alpha_{1}\left(\mu_{f \circ g}\left(\beta_{2}^{-1}(\log r)\right)\right)}=\infty .
$$

Similarly combining (3.5) of Case I and (3.10) of Case V we get that

$$
\liminf _{r \rightarrow+\infty} \frac{\alpha_{3}\left(\mu_{h \circ k}\left(\beta_{4}^{-1}(\log r)\right)\right)}{\alpha_{1}\left(\mu_{f \circ g}\left(\beta_{2}^{-1}(\log r)\right)\right)}=\infty .
$$

Analogously combining (3.6) of Case II and (3.9) of Case IV, we obtain that

$$
\lim _{r \rightarrow+\infty} \frac{\alpha_{3}\left(\mu_{h \circ k}\left(\beta_{4}^{-1}(\log r)\right)\right)}{\alpha_{1}\left(\mu_{f \circ g}\left(\beta_{2}^{-1}(\log r)\right)\right)}=\infty .
$$

Likewise combining (3.6) of Case II and (3.10) of Case V it follows that

$$
\lim _{r \rightarrow+\infty} \frac{\alpha_{3}\left(\mu_{h \circ k}\left(\beta_{4}^{-1}(\log r)\right)\right)}{\alpha_{1}\left(\mu_{f \circ g}\left(\beta_{2}^{-1}(\log r)\right)\right)}=\infty .
$$

Hence the first part of the theorem follows from (3.13), (3.14), (3.15) and (3.16).

Again combining (3.5) of Case I and (3.11) of Case VI we obtain for all sufficiently large values of $r$ that

$$
\frac{\exp \left(\alpha_{4}\left(\beta_{3}^{-1}\left(\alpha_{3}\left(\mu_{h \circ k}\left(\beta_{4}^{-1}(\log r)\right)\right)\right)\right)\right.}{\alpha_{1}\left(\mu_{f \circ g}\left(\beta_{2}^{-1}(\log r)\right)\right)} \geq \frac{r^{(1+o(1))\left(\lambda_{\left(\alpha_{4}, \beta_{4}\right)}[k]-\varepsilon\right)}}{C(1+o(1))\left(\rho_{\left(\alpha_{1}, \beta_{1}\right)}[f]+\varepsilon\right) r^{(1+o(1))\left(\rho_{\left(\alpha_{2}, \beta_{2}\right)}[g]+\varepsilon\right)}} .
$$

So from (3.12) and above we obtain that

$$
\lim _{r \rightarrow+\infty} \frac{\exp \left(\alpha_{4}\left(\beta_{3}^{-1}\left(\alpha_{3}\left(\mu_{h \circ k}\left(\beta_{4}^{-1}(\log r)\right)\right)\right)\right)\right)}{\alpha_{1}\left(\mu_{f \circ g}\left(\beta_{2}^{-1}(\log r)\right)\right)}=\infty .
$$

Similarly combining (3.6) of Case II and (3.11) of Case VI we also get the same conclusion. Therefore the second part of the theorem is established. 
Again combining (3.7) of Case III and (3.9) of Case IV it follows for all sufficiently large values of $r$ that

$$
\frac{\alpha_{3}\left(\mu_{h \circ k}\left(\beta_{4}^{-1}(\log r)\right)\right)}{\exp \left(\alpha_{2}\left(\beta_{1}^{-1}\left(\alpha_{1}\left(\mu_{f \circ g}\left(\beta_{2}^{-1}(\log r)\right)\right)\right)\right)\right.} \geq \frac{D(1+o(1))\left(\lambda_{\left(\alpha_{3}, \beta_{3}\right)}[h]-\varepsilon\right) r^{(1+o(1))\left(\lambda_{\left(\alpha_{4}, \beta_{4}\right)}[k]-\varepsilon\right)}}{r^{(1+o(1))\left(\rho_{\left(\alpha_{2}, \beta_{2}\right)}[g]+\varepsilon\right)}} .
$$

Now in view of (3.12) we obtain from (3.17) that

$$
\lim _{r \rightarrow+\infty} \frac{\alpha_{3}\left(\mu_{h \circ k}\left(\beta_{4}^{-1}(\log r)\right)\right)}{\exp \left(\alpha_{2}\left(\beta_{1}^{-1}\left(\alpha_{1}\left(\mu_{f \circ g}\left(\beta_{2}^{-1}(\log r)\right)\right)\right)\right)\right.}=\infty .
$$

Similarly combining (3.7) of Case III and (3.10) of Case V we get that

$$
\lim _{r \rightarrow+\infty} \frac{\alpha_{3}\left(\mu_{h \circ k}\left(\beta_{4}^{-1}(\log r)\right)\right)}{\exp \left(\alpha_{2}\left(\beta_{1}^{-1}\left(\alpha_{1}\left(\mu_{f \circ g}\left(\beta_{2}^{-1}(\log r)\right)\right)\right)\right)\right)}=\infty .
$$

Hence the third part of the theorem follows from (3.18) and (3.19).

Further combining (3.7) of Case III and (3.11) of Case VI we obtain for all sufficiently large values of $r$ that

$$
\frac{\exp \left(\alpha_{4}\left(\beta_{3}^{-1}\left(\alpha_{3}\left(\mu_{h \circ k}\left(\beta_{4}^{-1}(\log r)\right)\right)\right)\right)\right)}{\exp \left(\alpha_{2}\left(\beta_{1}^{-1}\left(\alpha_{1}\left(\mu_{f \circ g}\left(\beta_{2}^{-1}(\log r)\right)\right)\right)\right)\right.} \geq \frac{r^{(1+o(1))\left(\lambda_{\left(\alpha_{4}, \beta_{4}\right)}[k]-\varepsilon\right)}}{r^{(1+o(1))\left(\rho_{\left(\alpha_{2}, \beta_{2}\right)}[g]+\varepsilon\right)}} .
$$

Now in view of (3.12) we obtain from above that

$$
\lim _{r \rightarrow+\infty} \frac{\exp \left(\alpha_{4}\left(\beta_{3}^{-1}\left(\alpha_{3}\left(\mu_{h \circ k}\left(\beta_{4}^{-1}(\log r)\right)\right)\right)\right)\right)}{\exp \left(\alpha_{2}\left(\beta_{1}^{-1}\left(\alpha_{1}\left(\mu_{f \circ g}\left(\beta_{2}^{-1}(\log r)\right)\right)\right)\right)\right)}=\infty .
$$

This proves the fourth part of the theorem.

This implies the following theorem.

Theorem 8. Let $f$ and $g$ be any two entire functions such that

$$
\rho_{\left(\alpha_{1}, \beta_{1}\right)}[f \circ g]<\infty \quad \text { and } \quad \lambda_{\left(\alpha_{3}, \beta_{3}\right)}[g]>0 .
$$

Then

$$
\lim _{r \rightarrow \infty} \frac{\left\{\alpha_{1}\left(\mu_{f \circ g}\left(\beta_{1}^{-1}(\log r)\right)\right)\right\}^{2}}{\alpha_{3}\left(\mu_{g}\left(\beta_{3}^{-1}(\log r)\right)\right) \cdot \alpha_{3}\left(\mu_{g}\left(\beta_{3}^{-1}(r)\right)\right)}=0 .
$$

P r o o f. For arbitrary positive $\varepsilon$ we have for all sufficiently large values of $r$ that

$$
\alpha_{1}\left(\mu_{f \circ g}\left(\beta_{1}^{-1}(\log r)\right)\right) \leq\left(\rho_{\left(\alpha_{1}, \beta_{1}\right)}[f \circ g]+\varepsilon\right) \log r .
$$

Again for all sufficiently large values of $r$ we get

$$
\alpha_{3}\left(\mu_{g}\left(\beta_{3}^{-1}(\log r)\right)\right) \geq\left(\lambda_{\left(\alpha_{3}, \beta_{3}\right)}[g]-\varepsilon\right) \log r .
$$

Similarly for all sufficiently large values of $r$ we have

$$
\left(\alpha_{3}\left(\mu_{g}\left(\beta_{3}^{-1}(r)\right)\right)\right) \geq\left(\lambda_{\left(\alpha_{3}, \beta_{3}\right)}[g]-\varepsilon\right) r .
$$

From (3.20) and (3.21) we have for all sufficiently large values of $r$ that

$$
\frac{\alpha_{1}\left(\mu_{f \circ g}\left(\beta_{1}^{-1}(\log r)\right)\right)}{\alpha_{3}\left(\mu_{g}\left(\beta_{3}^{-1}(\log r)\right)\right)} \leq \frac{\left(\rho_{\left(\alpha_{1}, \beta_{1}\right)}[f \circ g]+\varepsilon\right) \log r}{\left(\lambda_{\left(\alpha_{3}, \beta_{3}\right)}[g]-\varepsilon\right) \log r} .
$$


As $\varepsilon>0$ is arbitrary we obtain from above that

$$
\limsup _{r \rightarrow+\infty} \frac{\alpha_{1}\left(\mu_{f \circ g}\left(\beta_{1}^{-1}(\log r)\right)\right)}{\alpha_{3}\left(\mu_{g}\left(\beta_{3}^{-1}(\log r)\right)\right)} \leq \frac{\rho_{\left(\alpha_{1}, \beta_{1}\right)}[f \circ g]}{\lambda_{\left(\alpha_{3}, \beta_{3}\right)}[g]} .
$$

Again from (3.20) and (3.22) we get for all sufficiently large values of $r$ that

$$
\frac{\alpha_{1}\left(\mu_{f \circ g}\left(\beta_{1}^{-1}(\log r)\right)\right)}{\alpha_{3}\left(\mu_{g}\left(\beta_{3}^{-1}(r)\right)\right)} \leq \frac{\left(\rho_{\left(\alpha_{1}, \beta_{1}\right)}[f \circ g]+\varepsilon\right) \log r}{\left(\lambda_{\left(\alpha_{3}, \beta_{3}\right)}[g]-\varepsilon\right) r} .
$$

Since $\varepsilon>0$ is arbitrary it follows from above that

$$
\lim _{r \rightarrow+\infty} \frac{\alpha_{1}\left(\mu_{f \circ g}\left(\beta_{1}^{-1}(\log r)\right)\right)}{\alpha_{3}\left(\mu_{g}\left(\beta_{3}^{-1}(r)\right)\right)}=0 .
$$

Thus the theorem follows from (3.23) and (3.24).

Remark 1. Theorem 1 to Theorem 8 can also be deduced in terms of maximum modulus of entire functions with the help of Lemma 1.

Theorem 9. Let $f$ and $g$ be any two entire functions such that

$$
\rho_{\left(\alpha_{2}, \beta_{2}\right)}[g]<\lambda_{\left(\alpha_{1}, \beta_{1}\right)}[f] \leq \rho_{\left(\alpha_{1}, \beta_{1}\right)}[f] .
$$

Also let $C$ be any positive constant and $\beta_{1} \in L_{2}$.

(i) Any one of the following two conditions are assumed to be satisfied:

(a) $\beta_{1}(r)=C\left(\exp \left(\alpha_{2}(r)\right)\right)$;

(b) $\exp \left(\alpha_{2}(r)\right)>\beta_{1}(r)$;

then

$$
\limsup _{r \rightarrow+\infty} \frac{\left\{\alpha_{1}\left(\mu_{f \circ g}\left(\beta_{2}^{-1}(\log r)\right)\right)\right\}^{2}}{\exp \left(\alpha_{1}\left(\mu_{f}\left(\beta_{1}^{-1}(\log r)\right)\right)\right) \cdot \beta_{1}\left(\mu_{g}\left(2 \beta_{2}^{-1}(\log r)\right)\right)}=0 .
$$

(ii) If $\alpha_{2}\left(\beta_{1}^{-1}(r)\right) \in L^{0}$, then

$$
\lim _{r \rightarrow+\infty} \frac{\exp \left(\alpha_{2}\left(\beta_{1}^{-1}\left(\alpha_{1}\left(\mu_{f \circ g}\left(\beta_{2}^{-1}(\log r)\right)\right)\right)\right) \cdot \alpha_{1}\left(\mu_{f \circ g}\left(\beta_{2}^{-1}(\log r)\right)\right)\right.}{\exp \left(\alpha_{1}\left(\mu_{f}\left(\beta_{1}^{-1}(\log r)\right)\right)\right) \cdot \beta_{1}\left(\mu_{g}\left(2 \beta_{2}^{-1}(\log r)\right)\right)}=0 .
$$

P r o o f. From the definition of generalized lower order $\left(\alpha_{1}, \beta_{1}\right)$ of $f$ we have for arbitrary positive $\varepsilon$ and for all sufficiently large values of $r$ that

$$
\exp \left(\alpha_{1}\left(\mu_{f}\left(\beta_{1}^{-1}(\log r)\right)\right)\right) \geq r^{\left(\lambda_{\left(\alpha_{1}, \beta_{1}\right)}[f]-\varepsilon\right)} .
$$

As $\rho_{\left(\alpha_{2}, \beta_{2}\right)}[g]<\lambda_{\left(\alpha_{1}, \beta_{1}\right)}[f]$ we can choose $\varepsilon>0$ in such a way that

$$
\rho_{\left(\alpha_{2}, \beta_{2}\right)}[g]+\varepsilon<\lambda_{\left(\alpha_{1}, \beta_{1}\right)}[f]-\varepsilon .
$$

Now combining (3.5) of Case I and (3.25) we have for all large positive numbers of $r$,

$$
\frac{\alpha_{1}\left(\mu_{f \circ g}\left(\beta_{2}^{-1}(\log r)\right)\right)}{\exp \left(\alpha_{1}\left(\mu_{f}\left(\beta_{1}^{-1}(\log r)\right)\right)\right)} \leq \frac{C(1+o(1))\left(\rho_{\left(\alpha_{1}, \beta_{1}\right)}[f]+\varepsilon\right) r^{(1+o(1))\left(\rho_{\left(\alpha_{2}, \beta_{2}\right)}[g]+\varepsilon\right)}}{r^{\left(\lambda_{\left(\alpha_{1}, \beta_{1}\right)}[f]-\varepsilon\right)}} .
$$


In view of (3.26) we get from above that

$$
\lim _{r \rightarrow+\infty} \frac{\alpha_{1}\left(\mu_{f \circ g}\left(\beta_{2}^{-1}(\log r)\right)\right)}{\exp \left(\alpha_{1}\left(\mu_{f}\left(\beta_{1}^{-1}(\log r)\right)\right)\right)}=0 .
$$

Again combining (3.6) of Case II and (3.25) we get for all sufficiently large positive numbers of $r$ that

$$
\frac{\alpha_{1}\left(\mu_{f \circ g}\left(\beta_{2}^{-1}(\log r)\right)\right)}{\exp \left(\alpha_{1}\left(\mu_{f}\left(\beta_{1}^{-1}(\log r)\right)\right)\right)} \leq \frac{(1+o(1))\left(\rho_{\left(\alpha_{1}, \beta_{1}\right)}[f]+\varepsilon\right) r^{(1+o(1))\left(\rho_{\left(\alpha_{2}, \beta_{2}\right)}[g]+\varepsilon\right)}}{r^{\left(\lambda_{\left(\alpha_{1}, \beta_{1}\right)}[f]-\varepsilon\right)}} .
$$

Now in view of (3.26) we obtain from above that

$$
\lim _{r \rightarrow+\infty} \frac{\alpha_{1}\left(\mu_{f \circ g}\left(\beta_{2}^{-1}(\log r)\right)\right)}{\exp \left(\alpha_{1}\left(\mu_{f}\left(\beta_{1}^{-1}(\log r)\right)\right)\right)}=0 .
$$

Further combining (3.7) of Case III and (3.25) we get for all sufficiently large positive numbers of $r$ that

$$
\frac{\exp \left(\alpha_{2}\left(\beta_{1}^{-1}\left(\alpha_{1}\left(\mu_{f \circ g}\left(\beta_{2}^{-1}(\log r)\right)\right)\right)\right)\right.}{\exp \left(\alpha_{1}\left(\mu_{f}\left(\beta_{1}^{-1}(\log r)\right)\right)\right)} \leq \frac{r^{(1+o(1))\left(\rho_{\left(\alpha_{2}, \beta_{2}\right)}[g]+\varepsilon\right)}}{r^{\left(\lambda_{\left(\alpha_{1}, \beta_{1}\right)}[f]-\varepsilon\right)}} .
$$

So in view of (3.26) we obtain from above that

$$
\lim _{r \rightarrow+\infty} \frac{\exp \left(\alpha_{2}\left(\beta_{1}^{-1}\left(\alpha_{1}\left(\mu_{f \circ g}\left(\beta_{2}^{-1}(\log r)\right)\right)\right)\right)\right)}{\exp \left(\alpha_{1}\left(\mu_{f}\left(\beta_{1}^{-1}(\log r)\right)\right)\right)}=0 .
$$

Now from (3.4) we get that

$$
\limsup _{r \rightarrow+\infty} \frac{\alpha_{1}\left(\mu_{f \circ g}\left(\beta_{2}^{-1}(\log r)\right)\right)}{\beta_{1}\left(\mu_{g}\left(2 \beta_{2}^{-1}(\log r)\right)\right)} \leq \rho_{\left(\alpha_{1}, \beta_{1}\right)}[f] .
$$

From (3.27) and (3.30) we obtain for all sufficiently large values of $r$ that

$$
\begin{gathered}
\limsup _{r \rightarrow+\infty} \frac{\left\{\alpha_{1}\left(\mu_{f \circ g}\left(\beta_{2}^{-1}(\log r)\right)\right)\right\}^{2}}{\exp \left(\alpha_{1}\left(\mu_{f}\left(\beta_{1}^{-1}(\log r)\right)\right)\right) \cdot \beta_{1}\left(\mu_{g}\left(2 \beta_{2}^{-1}(\log r)\right)\right)} \\
=\lim _{r \rightarrow+\infty} \frac{\alpha_{1}\left(\mu_{f \circ g}\left(\beta_{2}^{-1}(\log r)\right)\right)}{\exp \left(\alpha_{1}\left(\mu_{f}\left(\beta_{1}^{-1}(\log r)\right)\right)\right)} \cdot \limsup _{r \rightarrow+\infty} \frac{\alpha_{1}\left(\mu_{f \circ g}\left(\beta_{2}^{-1}(\log r)\right)\right)}{\beta_{1}\left(\mu_{g}\left(2 \beta_{2}^{-1}(\log r)\right)\right)} \leq 0 \cdot \rho_{\left(\alpha_{1}, \beta_{1}\right)}[f]=0 .
\end{gathered}
$$

Similarly from (3.28) and (3.30) we obtain that

$$
\limsup _{r \rightarrow+\infty} \frac{\left\{\alpha_{1}\left(\mu_{f \circ g}\left(\beta_{2}^{-1}(\log r)\right)\right)\right\}^{2}}{\exp \left(\alpha_{1}\left(\mu_{f}\left(\beta_{1}^{-1}(\log r)\right)\right)\right) \cdot \beta_{1}\left(\mu_{g}\left(2 \beta_{2}^{-1}(\log r)\right)\right)}=0 .
$$

Therefore the first part of the theorem follows from (3.31) and above.

Again from (3.29) and (3.30) we get for all large values of $r$ that

$$
\begin{gathered}
\limsup _{r \rightarrow+\infty} \frac{\exp \left(\alpha_{2}\left(\beta_{1}^{-1}\left(\alpha_{1}\left(\mu_{f \circ g}\left(\beta_{2}^{-1}(\log r)\right)\right)\right)\right) \cdot \alpha_{1}\left(\mu_{f \circ g}\left(\beta_{2}^{-1}(\log r)\right)\right)\right.}{\exp \left(\alpha_{1}\left(\mu_{f}\left(\beta_{1}^{-1}(\log r)\right)\right)\right) \cdot \beta_{1}\left(\mu_{g}\left(2 \beta_{2}^{-1}(\log r)\right)\right)} \\
=\lim _{r \rightarrow+\infty} \frac{\exp \left(\alpha_{2}\left(\beta_{1}^{-1}\left(\alpha_{1}\left(\mu_{f \circ g}\left(\beta_{2}^{-1}(\log r)\right)\right)\right)\right)\right.}{\exp \left(\alpha_{1}\left(\mu_{f}\left(\beta_{1}^{-1}(\log r)\right)\right)\right)} \cdot \limsup _{r \rightarrow+\infty} \frac{\alpha_{1}\left(\mu_{f \circ g}\left(\beta_{2}^{-1}(\log r)\right)\right)}{\beta_{1}\left(\mu_{g}\left(2 \beta_{2}^{-1}(\log r)\right)\right)} \leq 0 \cdot \rho_{\left(\alpha_{1}, \beta_{1}\right)}[f]=0, \\
\text { i.e., } \lim _{r \rightarrow+\infty} \frac{\exp \left(\alpha_{2}\left(\beta_{1}^{-1}\left(\alpha_{1}\left(\mu_{f \circ g}\left(\beta_{2}^{-1}(\log r)\right)\right)\right)\right) \cdot \alpha_{1}\left(\mu_{f \circ g}\left(\beta_{2}^{-1}(\log r)\right)\right)\right.}{\exp \left(\alpha_{1}\left(\mu_{f}\left(\beta_{1}^{-1}(\log r)\right)\right)\right) \cdot \beta_{1}\left(\mu_{g}\left(2 \beta_{2}^{-1}(\log r)\right)\right)}=0 .
\end{gathered}
$$

Thus the second part of the theorem is established.

In the line of Theorem 9 and with the help of Lemma 1, one can easily prove the following theorem and therefore its proof is omitted: 
Theorem 10. Let $f$ and $g$ be any two entire functions such that

$$
\rho_{\left(\alpha_{2}, \beta_{2}\right)}[g]<\lambda_{\left(\alpha_{1}, \beta_{1}\right)}[f] \leq \rho_{\left(\alpha_{1}, \beta_{1}\right)}[f] .
$$

Also let $C$ be any positive constant and $\beta_{1} \in L_{2}$.

(i) Any one of the following two conditions are assumed to be satisfied:

(a) $\beta_{1}(r)=C\left(\exp \left(\alpha_{2}(r)\right)\right)$;

(b) $\exp \left(\alpha_{2}(r)\right)>\beta_{1}(r)$;

then

$$
\limsup _{r \rightarrow+\infty} \frac{\left\{\alpha_{1}\left(M_{f \circ g}\left(\beta_{2}^{-1}(\log r)\right)\right)\right\}^{2}}{\exp \left(\alpha_{1}\left(M_{f}\left(\beta_{1}^{-1}(\log r)\right)\right)\right) \cdot \beta_{1}\left(M_{g}\left(\beta_{2}^{-1}(\log r)\right)\right)}=0 .
$$

(ii) If $\alpha_{2}\left(\beta_{1}^{-1}(r)\right) \in L^{0}$, then

$$
\lim _{r \rightarrow+\infty} \frac{\exp \left(\alpha_{2}\left(\beta_{1}^{-1}\left(\alpha_{1}\left(M_{f \circ g}\left(\beta_{2}^{-1}(\log r)\right)\right)\right)\right)\right) \cdot \alpha_{1}\left(M_{f \circ g}\left(\beta_{2}^{-1}(\log r)\right)\right)}{\exp \left(\alpha_{1}\left(M_{f}\left(\beta_{1}^{-1}(\log r)\right)\right)\right) \cdot \beta_{1}\left(M_{g}\left(\beta_{2}^{-1}(\log r)\right)\right)}=0 .
$$

Theorem 11. Let $f$ and $g$ be any two entire functions such that

$$
\lambda_{\left(\alpha_{1}, \beta_{1}\right)}[f]<\infty \quad \text { and } \quad \rho_{\left(\alpha_{3}, \beta_{3}\right)}[f \circ g]<\infty
$$

where $\alpha_{2}, \beta_{1} \in L_{2}$. Then

$$
\limsup _{r \rightarrow+\infty} \frac{\alpha_{1}\left(\mu_{f \circ g}\left(\beta_{2}^{-1}(\log r)\right)\right) \cdot \alpha_{3}\left(\mu_{f \circ g}\left(\beta_{3}^{-1}(r)\right)\right)}{\beta_{1}\left(\mu_{g}\left(2 \beta_{2}^{-1}(\log r)\right)\right) \cdot \alpha_{2}\left(\mu_{g}\left(\beta_{2}^{-1}(r)\right)\right)} \leq \frac{\rho_{\left(\alpha_{3}, \beta_{3}\right)}[f \circ g] \cdot \rho_{\left(\alpha_{1}, \beta_{1}\right)}[f]}{\lambda_{\left(\alpha_{2}, \beta_{2}\right)}[g]} .
$$

$\mathrm{P}$ r o o f. For all sufficiently large values of $r$ we have

$$
\alpha_{3}\left(\mu_{f \circ g}\left(\beta_{3}^{-1}(r)\right)\right) \leq\left(\rho_{\left(\alpha_{3}, \beta_{3}\right)}[f \circ g]+\varepsilon\right) r .
$$

Again for all sufficiently large values of $r$ it follows that

$$
\alpha_{2}\left(\mu_{g}\left(\beta_{2}^{-1}(r)\right)\right) \geq\left(\lambda_{\left(\alpha_{2}, \beta_{2}\right)}[g]-\varepsilon\right) r .
$$

Now combining (3.32) and (3.33) we have for all sufficiently large values of $r$ that

$$
\frac{\alpha_{3}\left(\mu_{f \circ g}\left(\beta_{3}^{-1}(r)\right)\right)}{\alpha_{2}\left(\mu_{g}\left(\beta_{2}^{-1}(r)\right)\right)} \leq \frac{\rho_{\left(\alpha_{3}, \beta_{3}\right)}[f \circ g]+\varepsilon}{\lambda_{\left(\alpha_{2}, \beta_{2}\right)}[g]-\varepsilon} .
$$

As $\varepsilon>0$ is arbitrary we get from above that

$$
\limsup _{r \rightarrow+\infty} \frac{\alpha_{3}\left(\mu_{f \circ g}\left(\beta_{3}^{-1}(r)\right)\right)}{\alpha_{2}\left(\mu_{g}\left(\beta_{2}^{-1}(r)\right)\right)} \leq \frac{\rho_{\left(\alpha_{3}, \beta_{3}\right)}[f \circ g]}{\lambda_{\left(\alpha_{2}, \beta_{2}\right)}[g]} .
$$

Now from (3.30) and (3.34) we obtain that

$$
\begin{gathered}
\limsup _{r \rightarrow+\infty} \frac{\alpha_{1}\left(\mu_{f \circ g}\left(\beta_{2}^{-1}(\log r)\right)\right) \cdot \alpha_{3}\left(\mu_{f \circ g}\left(\beta_{3}^{-1}(r)\right)\right)}{\beta_{1}\left(\mu_{g}\left(2 \beta_{2}^{-1}(\log r)\right)\right) \cdot \alpha_{2}\left(\mu_{g}\left(\beta_{2}^{-1}(r)\right)\right)} \\
\leq \limsup _{r \rightarrow+\infty} \frac{\alpha_{1}\left(\mu_{f \circ g}\left(\beta_{2}^{-1}(\log r)\right)\right)}{\beta_{1}\left(\mu_{g}\left(2 \beta_{2}^{-1}(\log r)\right)\right)} \cdot \limsup _{r \rightarrow+\infty} \frac{\alpha_{3}\left(\mu_{f \circ g}\left(\beta_{3}^{-1}(r)\right)\right)}{\alpha_{2}\left(\mu_{g}\left(\beta_{2}^{-1}(r)\right)\right)} \leq \frac{\rho_{\left(\alpha_{3}, \beta_{3}\right)}[f \circ g] \cdot \rho_{\left(\alpha_{1}, \beta_{1}\right)}[f]}{\lambda_{\left(\alpha_{2}, \beta_{2}\right)}[g]} .
\end{gathered}
$$

Hence the theorem follows.

In the line of Theorem 11 and with the help of Lemma 1, one can easily proof the following theorem and therefore its proof is omitted: 
Theorem 12. Let $f$ and $g$ be any two entire functions such that

$$
\lambda_{\left(\alpha_{1}, \beta_{1}\right)}[f]<\infty \quad \text { and } \quad \rho_{\left(\alpha_{3}, \beta_{3}\right)}[f \circ g]<\infty
$$

where $\alpha_{2}, \beta_{1} \in L_{2}$. Then

$$
\limsup _{r \rightarrow+\infty} \frac{\alpha_{1}\left(M_{f \circ g}\left(\beta_{2}^{-1}(\log r)\right)\right) \cdot \alpha_{3}\left(M_{f \circ g}\left(\beta_{3}^{-1}(r)\right)\right)}{\beta_{1}\left(M_{g}\left(\beta_{2}^{-1}(\log r)\right)\right) \cdot \alpha_{2}\left(M_{g}\left(\beta_{2}^{-1}(r)\right)\right)} \leq \frac{\rho_{\left(\alpha_{3}, \beta_{3}\right)}[f \circ g] \cdot \rho_{\left(\alpha_{1}, \beta_{1}\right)}[f]}{\lambda_{\left(\alpha_{2}, \beta_{2}\right)}[g]} .
$$

Theorem 13. Let $f$ and $g$ be any two entire functions such that

$$
\rho_{\left(\alpha_{1}, \beta_{1}\right)}[f]<\infty \quad \text { and } \quad \lambda_{\left(\alpha_{3}, \beta_{3}\right)}[f \circ g]=\infty .
$$

Then

$$
\lim _{r \rightarrow+\infty} \frac{\alpha_{3}\left(\mu_{f \circ g}(r)\right)}{\alpha_{1}\left(\mu_{f}\left(\beta_{1}^{-1}\left(\beta_{3}(r)\right)\right)\right)}=\infty
$$

P r o o f. Let us suppose that the conclusion of the theorem do not hold. Then we can find a constant $\Delta>0$ such that for a sequence of values of $r$ tending to infinity

$$
\alpha_{3}\left(\mu_{f \circ g}(r)\right) \leq \Delta \cdot \alpha_{1}\left(\mu_{f}\left(\beta_{1}^{-1}\left(\beta_{3}(r)\right)\right)\right) .
$$

Again from the definition of $\rho_{\left(\alpha_{1}, \beta_{1}\right)}[f]$, it follows for all sufficiently large values of $r$ that

$$
\alpha_{1}\left(\mu_{f}\left(\beta_{1}^{-1}\left(\beta_{3}(r)\right)\right)\right) \leq\left(\rho_{\left(\alpha_{1}, \beta_{1}\right)}[f]+\epsilon\right) \beta_{3}(r) .
$$

Thus from (3.35) and (3.36), we have for a sequence of values of $r$ tending to infinity that

$$
\begin{gathered}
\alpha_{3}\left(\mu_{f \circ g}(r)\right) \leq \Delta\left(\rho_{\left(\alpha_{1}, \beta_{1}\right)}[f]+\epsilon\right) \beta_{3}(r) \\
\text { i.e., } \frac{\alpha_{3}\left(\mu_{f \circ g}(r)\right)}{\beta_{3}(r)} \leq \frac{\Delta\left(\rho_{\left(\alpha_{1}, \beta_{1}\right)}[f]+\epsilon\right) \beta_{3}(r)}{\beta_{3}(r)} \\
\text { i.e., } \liminf _{r+\infty} \frac{\alpha_{3}\left(\mu_{f \circ g}(r)\right)}{\beta_{3}(r)}=\lambda_{\left(\alpha_{3}, \beta_{3}\right)}[f \circ g]<\infty .
\end{gathered}
$$

This is a contradiction. Thus the theorem follows.

Remark 2. Theorem 13 is also valid with "limit superior" instead of "limit" if $\lambda_{\left(\alpha_{3}, \beta_{3}\right)}[f \circ g]=\infty$ is replaced by $\rho_{\left(\alpha_{3}, \beta_{3}\right)}[f \circ g]=\infty$ while the other conditions remain the same.

Analogously one may also state the following theorem without its proof as it may be carried out in the line of Theorem 13.

Theorem 14. Let $f$ and $g$ be any two entire functions such that

$$
\rho_{\left(\alpha_{1}, \beta_{1}\right)}[g]<\infty \quad \text { and } \quad \rho_{\left(\alpha_{3}, \beta_{3}\right)}[f \circ g]=\infty .
$$

Then

$$
\limsup _{r \rightarrow+\infty} \frac{\alpha_{3}\left(\mu_{f \circ g}(r)\right)}{\alpha_{1}\left(\mu_{g}\left(\beta_{1}^{-1}\left(\beta_{3}(r)\right)\right)\right)}=\infty
$$

Remark 3. Theorem 14 is also valid with "limit" instead of "limit superior" if $\rho_{\left(\alpha_{3}, \beta_{3}\right)}[f \circ g]=\infty$ is replaced by $\lambda_{\left(\alpha_{3}, \beta_{3}\right)}[f \circ g]=\infty$ and the other conditions remain the same.

Remark 4. Theorem 13, Theorem 14, Remark 2 and Remark 3 can also be deduced in terms of maximum modulus of entire functions. 


\section{Conclusion}

Actually this paper deals with the extension of the researches on the growth properties of composite entire functions on the basis of their generalized order $(\alpha, \beta)$ where $\alpha$ and $\beta$ are continuous non-negative functions on $(-\infty,+\infty)$. This assumption can also be modified by the treatment of the ideas of generalized type $(\alpha, \beta)$. Moreover, some extensions of the same may be done in the light of generalized relative order $(\alpha, \beta)$. Furthermore, the concept of generalized order $(\alpha, \beta)$ and generalized type $(\alpha, \beta)$ should have a broad range of applications in complex dynamics, factorization theory of entire functions of single complex variable, the solution of complex differential equations etc. which may be an ample scope of further research.

\section{Acknowledgements}

The authors are thankful to the referee for his / her valuable suggestions towards the improvement of the paper.

\section{REFERENCES}

1. Biswas T. On some inequalities concerning relative $(p, q)-\varphi$ type and relative $(p, q)-\varphi$ weak type of entire or meromorphic functions with respect to an entire function. J. Class. Anal., 2018. Vol. 13, No. 2. P. 107-122. DOI: $10.7153 /$ jca-2018-13-07

2. Biswas T., Biswas C., Biswas R. A note on generalized growth analysis of composite entire functions. Poincare J. Anal. Appl., 2020. Vol. 7, No. 2. P. 277-286.

3. Chyzhykov I., Semochko N. Fast growing entire solutions of linear differential equations. Math. Bull. Shevchenko Sci. Soc., 2016. Vol. 13. P. 68-83. http://journals.iapmm.lviv.ua/ojs/index.php/MBSSS/article/viewFile/2107/2501

4. Clunie J. The composition of entire and meromorphic functions. In: Mathematical Essays dedicated to A.J. Macintyre. Hari Shankar (ed.) Ohio: Ohio University Press, 1970. P. 75-92.

5. Juneja O.P., Kapoor G. P., Bajpai S. K. On the $(p, q)$-order and lower $(p, q)$-order of an entire function. J. Reine Angew. Math., 1976. Vol. 282. P. 53-67. DOI: 10.1515/crll.1976.282.53

6. Shen X., Tu J., Xu H.Y. Complex oscillation of a second-order linear differential equation with entire coefficients of $[p, q]-\varphi$ order. Adv. Differ. Equ., 2014. Vol. 2014, No. 1. Art. no. 200. 14 p. DOI: $10.1186 / 1687-1847-2014-200$

7. Sheremeta M. N. Connection between the growth of the maximum of the modulus of an entire function and the moduli of the coefficients of its power series expansion. Izv. Vyssh. Uchebn. Zaved Mat., 1967. Vol. 2. P. 100-108. (in Russian)

8. Sato D. On the rate of growth of entire functions of fast growth. Bull. Amer. Math. Soc., 1963. Vol. 69, No. 3. P. 411-414. https://projecteuclid.org/euclid.bams/1183525273

9. Singh A. P. On maximum term of composition of entire functions. Proc. Nat. Acad. Sci. India Sect. A, 1989. Vol. 59, Part I. P. 103-115.

10. Singh A.P., Baloria M.S. On the maximum modulus and maximum term of composition of entire functions. Indian J. Pure Appl. Math., 1991. Vol. 22, No. 12. P. 989-996. https://insa.nic.in/writereaddata/UpLoadedFiles/IJPAM/20005a1f_989.pdf

11. Valiron G. Lectures on the General Theory of Integral Functions. NY: Chelsea Publishing Company, 1949. $234 \mathrm{p}$.

12. Yang L. Value Distribution Theory. Berlin, Heidelberg: Springer-Verlag, 1993. DOI: $10.1007 / 978-3-662-02915-2$ 